\title{
Delimitacion Del Periodo Critico de Competencia de Malezas en EL CULTIVo de LINo (Linum usitatissimum) ${ }^{1}$
}

\author{
Determination of the Critical Period of Weed Competition in Linseed (Linum usitatissimum)
}

BARREYRO, R.A. ${ }^{2}$ y SÁNCHEZ VALLDUVI, G.E. ${ }^{2}$

\begin{abstract}
RESUMEN - La planta de lino oleaginoso es especialmente sensible a la competencia con malezas, que limita el rendimiento potencial. El objetivo de este trabajo fue delimitar el período crítico de competencia del lino con una comunidad natural de malezas. Se realizó una experiencia de campo durante 1993, 1994 y 1995 en La Plata, Argentina. Los tratamientos consistieron en mantener el lino con y sin presencia de malezas en distintas etapas de su desarrollo. La comunidad de malezas fue evaluada en su composición y producción de materia seca y el cultivo en su rendimiento en semillas y componentes. El período crítico se delimitó entre los 30 y 80 días después de la siembra. En este período, la disminución de rendimiento de lino respecto del testigo sin malezas fue superior al 10\%. La disminución de rendimiento del cultivo totalmente enmalezado fue del $79 \%$ respecto del desmalezado todo el ciclo. Fue afectado el número de semillas por planta ya que su definición está incluida en el período delimitado. Los resultados obtenidos sugieren que las alternativas de control deben considerar el período crítico y también propiciar su utilización en modelos de predicción.
\end{abstract}

Palabras clave: competencia, rendimiento, comunidad de malezas, semillas por planta.

\begin{abstract}
Linseed plant is especially sensitive to weed competition, which limits its potential yield. The aim of this work was to determine the critical period of linseed competition with a natural weed community. A field experiment was carried out in 1993, 1994 and 1995 in La Plata, Argentina. Treatments consisted of keeping linseed with and without weeds at different stages of development. Weed composition and dry biomass, and linseed yield and its components were evaluated. The critical period of competition was determined, starting at 30 days and ending 80 days after crop seeding. Linseed yield decrease was greater than 10\% during the critical period of competition, when compared to the check plot kept without competition. The maximum yield decrease of the check plot kept totally weeded crop from seeding to harvest was $79 \%$. The number of seeds per linseed plant was affected, since its definition was included in the delimited critical period. The results obtained suggest that alternative weed management must consider long critical periods and should be used in predictive models.
\end{abstract}

Key words: alternative management, predictive models, weed community, seeds per plant.

\section{INTRODUCCION}

El lino produce aceite industrial para el mercado mundial, siendo Argentina uno de los principales países exportadores. Constituye una alternativa de producción entre los cereales de invierno tradicionales como el trigo y actualmente su área de siembra se concentra en las provincias de Entre Ríos y Buenos Aires.

Si bien existe un importante avance en la mejora genética, la tecnología de producción utilizada presenta deficiencias, dentro de las cuales se destaca el manejo de las malezas (Acosta, 1988). Se ha señalado que es un cultivo

\footnotetext{
Recebido para publicação em 29/6/2000 e na forma revisada em 9/12/2002.

2 Profesor adjunto y jefe de Trabajos prácticos respectivamente del Curso de Cultivos industriales. Departamento de Producción vegetal. Facultad de ciencias agrarias y forestales. Universidad Nacional de La Plata. CC 31 (1900) La Plata. Argentina.
} 
especialmente sensible a la competencia interespecífica con mayor susceptibilidad que la cebada, la colza y el trigo (O’Donovan \& Sharma, 1983). Las mayores disminuciones de rendimiento aparecen al aumentar el período de competencia, siendo el componente más afectado el número de semillas por planta (Bell \& Nalewaja 1968; Brasesco, 1969; Gruenhagen \& Nalewaja, 1969; O'Donovan \& Sharma, 1983; Barreyro et al. 1994 y Sánchez Vallduví et al. 1997). Presenta mayor sensibilidad en las primeras etapas de desarrollo, disminuyendo la misma cuando la presencia de malezas continúa después de la aparición de los primeros botones florales (Sánchez Vallduví et al. 1998a).

El manejo de malezas es una herramienta de valor para incrementar los rendimientos y realizar un mejor aprovechamiento de los recursos. Es necesario conocer los factores que interactúan al establecerse la competencia, entre los que se destacan el período de mayor susceptibilidad (Brasesco, 1969), las poblaciones de malezas (Pletzer \& Falcon, 1991), la dinámica de crecimiento del cultivo (Sánchez Vallduví et al., 1998b) y la magnitud de las pérdidas de rendimiento registradas.

La delimitación del período crítico de competencia es un dato propio de cada ambiente agroecológico que permite la planificación del manejo de malezas en el cultivo (Aldrich \& Kremer, 1997; Radosevich et al. 1997). En Argentina, se ha delimitado este período en soja (Cholaky et al. 1984), algodonero (Pletzer, 1986), coriandro (García et al. 1996) y arroz (de la Vega et al., 1997), pero no se había establecido para el lino.

La determinación del período crítico en el cultivo de lino constituye una herramienta para disminuir los daños por enmalezamiento, aportando elementos tanto para evaluar la conveniencia de utilizar diferentes alternativas de manejo y control, como para elaborar modelos de competencia.

El objetivo del presente trabajo fue delimitar el período crítico de competencia interespecífica del cultivo de lino con una comunidad natural de malezas.

\section{MATERIAL Y METODOS}

Se realizaron ensayos de campo durante los años 1993, 1994 y 1995 en la Estación experimental Julio Hirschhorn, de la Universidad Nacional de La Plata, Argentina ( $34^{\circ} 52^{\prime} \mathrm{L}$ S, altura snm $15 \mathrm{~m}$ ).

Las experiencias se realizaron sobre suelos Argiudol típico. Los tratamientos consistieron en desmalezar y mantener enmalezado el cultivo de lino durante diferentes períodos, dando lugar a los siguientes tratamientos:

DTC: Desmalezado todo el ciclo.

DFF: Desmalezado hasta fin de la floración.

DBV: Desmalezado hasta los botones florales visibles.

D 10: Desmalezado hasta $10 \mathrm{~cm}$ de altura del lino.

E 10: Enmalezado hasta los $10 \mathrm{~cm}$ de altura del lino.

EBV: Enmalezado hasta los botones florales visibles.

EFF: Enmalezado hasta fin de la floración.

ETC: Enmalezado todo el ciclo.

Se utilizaron parcelas de $1,4 \mathrm{~m}$ por $7,5 \mathrm{~m}$, sembradas a $0,20 \mathrm{~m}$ entre líneas dispuestas en un diseño en bloques al azar con cuatro repeticiones. El laboreo del suelo comenzó en abril, sembrándose las variedades Areco INTA (13-7-93), Rojas INTA (6-7-94) y Tape INTA (5-7-95) . La densidad de siembra utilizada en todos los casos fue de $60 \mathrm{~kg} \mathrm{ha}^{-1}$ de semilla, obteniéndose un promedio de 202, 325 y $282 \mathrm{pl} \mathrm{m}^{-2}$ para 1993, 94 y 95 respectivamente.

Los desmalezados se efectuaron mecánicamente. En el momento de la remoción de malezas se tomó una muestra al azar de $0,5 \mathrm{~m}^{2}$ de biomasa aérea, separando lino y malezas en cada parcela. Se identificaron las malezas predominantes, se secaron en estufa a $60{ }^{\circ} \mathrm{C}$ hasta peso constante y se registró el peso seco.

Al alcanzar la madurez comercial se cosechó $0,3 \mathrm{~m}^{2}$ de cada parcela, se registró el número de plantas por metro cuadrado, ser rendimiento en semilla, el peso de 1000 semillas y se calculó el número de semillas por planta. Los resultados obtenidos se sometieron al análisis de varianza y para la comparación de medias se usó la prueba de Tukey al 0,05 de probabilidades. La delimitación del período crítico se realizó utilizando valores promedios de rendimientos relativos (porcentaje de rendimiento respecto del testigo DTC). Se delimitó como el lapso en el cual el rendimiento presentó una disminución mayor al $10 \%$ respecto del tratamiento DTC (de la Vega et al. 1997). 
En la Figura 1 se presentan los promedios de la temperatura media mensual $\left({ }^{\circ} \mathrm{C}\right)$ y las lluvias mensuales $(\mathrm{mm})$ ocurridas en el barbecho y el ciclo del cultivo para los tres años de ensayo.

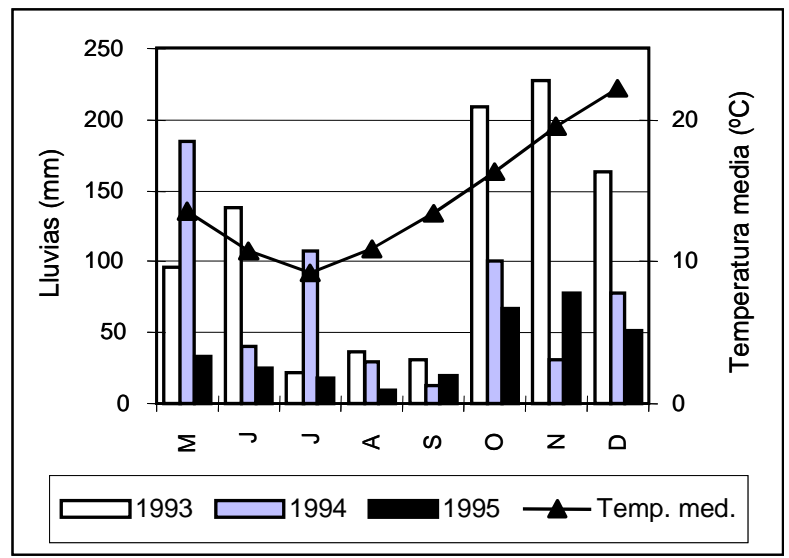

Figura 1 - Promedio de temperatura media mensual y lluvias mensuales registradas durante el barbecho y ciclo del cultivo. La Plata. 1993-1994-1995.

Se registraron las etapas fenológicas del cultivo, y al no presentar desvíos significativos, se promediaron para los tres años los valores de días transcurridos desde la siembra hasta distintas etapas, obteniéndose los siguientes valores: $10 \mathrm{~cm}$ de altura del lino (70 días), primeros botones florales visibles (BFV- 98 días), fin de floración (FF-126 días) y cosecha (COS-174 días).

\section{RESULTADOS Y DISCUSION}

La composición de la comunidad de malezas para los tres años fue similar y representativa para las condiciones del cultivo en Argentina (Pletzer \& Falcon, 1991). Las especies predominantes fueron: Lolium multiflorum, Avena fatua, Setaria verticilata, Bromus unioloides, Briza minor, Brassica campestris, Brassica napus, Raphanus sativus, Stellaria media, Nasturtium officinalis, Spergula arvesis, Carduus acanthoides, Anthemis cotula, Chenopodium album, Lamium amplexicaule, Convolvulus arvensis, Polygonum aviculre, Ammi majus.

La producción de biomasa de malezas siguió la tendencia marcada por la cantidad de lluvias para cada año, así en el año de mayor registro de lluvias durante todo el ciclo (1993) se produjo la mayor biomasa total de malezas (Figuras 1 y 2). En los tres años evaluados las pérdidas de rendimiento por ETC (enmalezamiento total) fueron de 63, 54 y 79\% para 1993, 1994 y 1995 respectivamente.

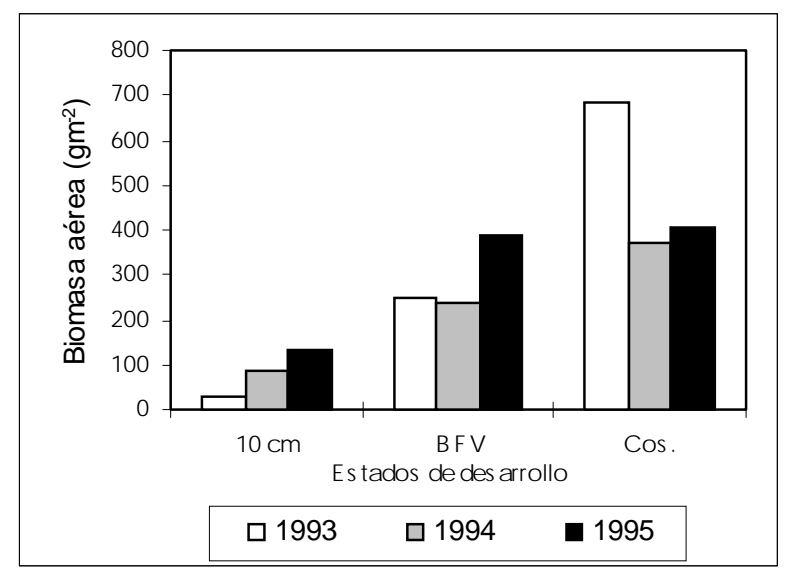

Figura 2 - Producción de biomasa de malezas. La Plata. 19931994-1995.

Para las condiciones de la experiencia, se delimitó el período crítico de competencia entre los 30 y 80 días desde la siembra (Figura 3). Este período es más amplio que en otros cultivos estudiados como soja (Cholaky et al. 1984), algodonero (Pletzer, 1986), coriandro (García et al. 1996) y arroz (de la Vega et al. 1997). Este comportamiento coincide con el largo período de alta sensibilidad al enmalezamiento del lino (O' Donovan \& Sharma, 1983). Asimismo, se observó que la merma de rendimiento de cada año se relacionó con la biomasa de malezas acumulada en botones florales visibles, estado en el cual finaliza el período crítico de competencia.

El único componente de rendimiento afectado por la competencia fue el número de semillas por planta lo que coincide con Bell \& Nalewaja (1968); Brasesco (1969) Gruenhagen \& Nalewaja (1969); Barreyro et al. (1994) y Sánchez Vallduví et al. (1997).

Se observó que, dentro del período crítico de competencia disminuyeron significativamente los valores de semillas por planta. En 1995, las malezas provocaron mayores pérdidas porcentuales, de las semillas por planta. 
Se detectó también que el efecto competitivo sobre este componente comenzó más temprano (Figura 4c). En 1993 y 1994 (Figuras 4a y 4b) el comienzo de la declinación de los valores de semillas por planta se produjo más tardíamente presentando en ambos años menores valores de biomasa de malezas en botones florales visibles que en 1995 (Figura 3).

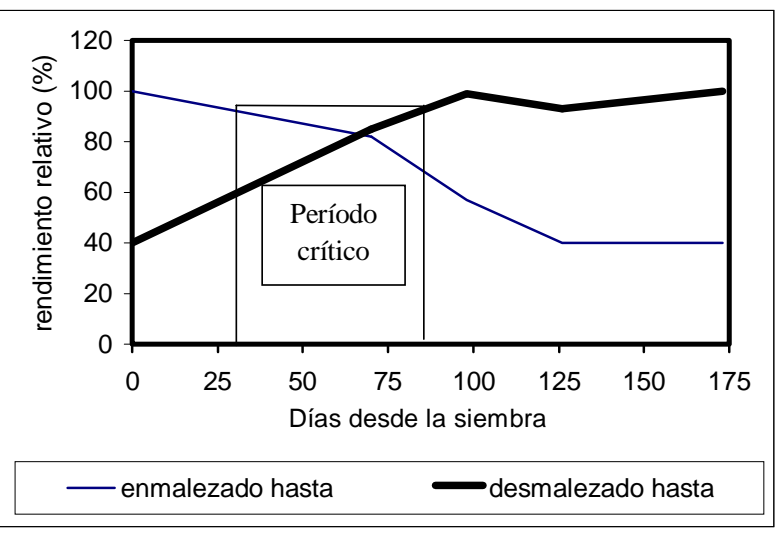

Figura 3 - Delimitación del período crítico de competencia interespecífica en el cultivo de lino La Plata. 1993-19941995.

Tabla 1 - Efectos del enmalezamiento en el cultivo de lino sobre rendimiento en semillas. La Plata. Argentina. 1993, 1994 y 1995

\begin{tabular}{|c|c|c|c|}
\hline Tratamiento & \multicolumn{3}{|c|}{ Rendimiento $\left(\mathrm{g} \mathrm{m}^{-2}\right)$} \\
\hline Años & 93 & 94 & 95 \\
\hline DTC & $218 \mathrm{a}$ & $195 \mathrm{a}$ & $325 \mathrm{a}$ \\
\hline DFF & $201 \mathrm{a}$ & $192 \mathrm{a}$ & $274 \mathrm{a}$ \\
\hline D PP & $218 \mathrm{a}$ & $198 \mathrm{a}$ & $295 \mathrm{a}$ \\
\hline D 10 & $188 \mathrm{a}$ & $162 \mathrm{a}$ & $282 \mathrm{a}$ \\
\hline E 10 & $176 \mathrm{~b}$ & $167 \mathrm{a}$ & $243 \mathrm{ab}$ \\
\hline E PP & $121 \mathrm{c}$ & $119 \mathrm{bc}$ & $156 \mathrm{bc}$ \\
\hline EFF & $85 \mathrm{~d}$ & $86 \mathrm{c}$ & $79 \mathrm{c}$ \\
\hline ETC & $81 \mathrm{~d}$ & $91 \mathrm{c}$ & $74 \mathrm{c}$ \\
\hline
\end{tabular}

Los valores dentro de la misma columna, seguidos por la misma letra no difieren entre sí al nivel de 0,05 de probabilidades según la prueba de Tukey.

La extensa duración del período crítico delimitado y la magnitud de las pérdidas de rendimiento registrada indican la necesidad de planear cuidadosamente el manejo de las malezas antes y durante dicha etapa. Resulta necesario también realizar una adecuada selección de las alternativas a utilizar, tendiente a un aprovechamiento racional de los recursos.
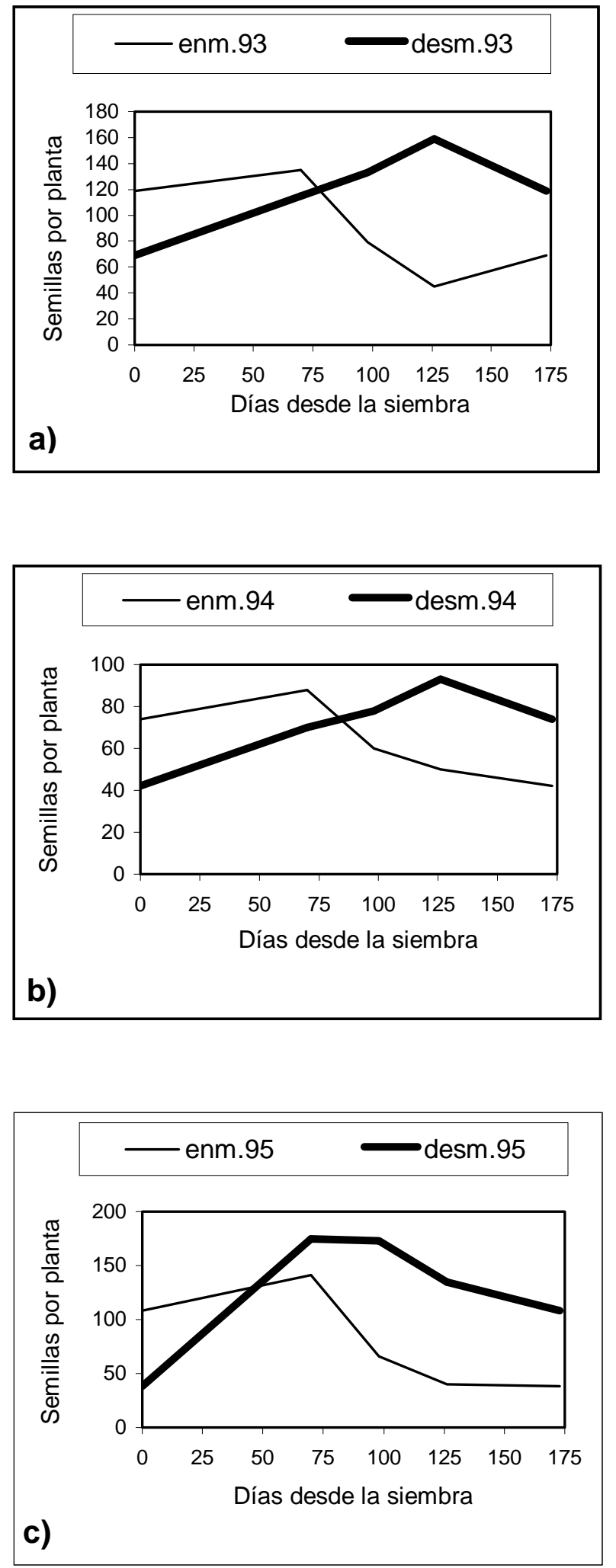

Figura 4 - Número de semillas por planta ante distintas etapas de enmalezamiento y desmalezamiento. La Plata. 1993(a), 1994(b), 1995(c). 


\section{LITERATURA CITADA}

ACOSTA, P. P. Por qué no se incrementa el rendimiento linero argentino? Pergamino: EEA/INTA, 1988. $11 \mathrm{p}$. (Divulgación Técnica, 71).

ALDRICH, R. J.; KREMER, R. J. Competitiveness of weeds. In: ALDRICH, R. J.; KREMER, R. J. Principles in weed management. Ames: Iowa State University Press/ Ames, 1997. p.15-34.

BARREYRO, R. A. et al. Período crítico de competencia interespecífica en el cultivo de lino (Linum usitatissimun L). Oleaginosos, v. 9, p. 10-14, 1994.

BELL, A. R.; NALEWAJA, J. D. Effect of duration of wild oat competition in flax. Weed Sci., v. 16, p. 509-512, 1968.

BRASESCO, J. A. I. Influencia de las malezas sobre los rendimientos de lino en Paraná. Entre Ríos: INTA 1969. 16 p. (Série Técnica, 25)

CHOLAKY, L.; VALETTI, O.; PIATTI, F. Período crítico de competencia de comunidades naturales de malezas en cultivo de soja (Glycine max L.) de hábito determinado. R. Univers. Nacional de Río Cuarto, v. 2, p. 219-230, 1984.

DE LA VEGA, M. H. et al. Período crítico de competencia de malezas en arroz (Oryza sativa $\mathrm{L}$.) de secano en Tucumán. In: CONGRESO LATINOAMERICANO DE MALEZAS, 13., 1997, Buenos Aires. Acta I, Buenos Aires: 1997. p. 13-17.

GARCIA, M. et al. Determinación del período de competencia de las malezas en el cultivo de coriandro (Coriandrum sativum L.). An. Soc. Argentina Invest. Prod. Arom., v. 14, p. 255-267, 1996.
GRUENHAGEN, R. D.; NALEWAJA, J. D. Competition between flax and wild buckwheat. Weed Sci., v. 17, p. 380-384, 1969.

O'DONOVAN, J.T.; SHARMA, M.P. Wild oats, competition and crop losses. In: WILD DAT/SYMPOSIUM, 1983, Canadá. Proceedings... Canadá: 1983. p. 27-42.

PLETZER, H. F. Interferencia de malezas en el cultivo de algodón. Roque Saenz Peña: EEA/INTA, 1986. 27 p. (Boletin, 97).

PLETZER, H. F.; FALCON, L. M. Las malezas en el cultivo de lino en el oeste entrerriano. Paraná, Argentina: EEA/INTA, 1991. 15 p. (Série técnica, 56)

RADOSEVICH, S.; HOLT, J.; GHERSA, C. Associations of weeds and crops. In: Weed ecology implications for management. Inc. NY: John WIley \& Sons, 1997. p. 201209.

SÁNCHEZ VALLDUVI, G. E.; MANGHI, M. V.; BARREYRO, R. A. Efecto de la presencia de malezas en distintos períodos del cultivo de lino oleaginoso (Linum usitatissimun L.). Agro-Ciencia, v. 13, n. 3, p. 257-263, 1997.

SÁNCHEZ VALLDUVI, G. E.; FLORES, C.; MANGHI, M. V. Acumulación y partición de materia seca aérea y radical en el cultivo de lino (Linum ussitatisimun L.). In: REUNION NACIONAL DE OLEAGINOSAS, 3., 1998, Bahía Blanca. Reunion... Bahía Blanca: 1998a. p. 219-220.

SÁNCHEZ VALLDUVI, G. E. et al. Efecto de la competencia interespecífica en distintas etapas ontogénicas del lino oleaginoso (Linum usitatissimun L.). In:

CONGRESO LATIONOAMERICANO DE MALEZAS, 13., 1998, Buenos Aires, Acta II... Buenos Aires: 1998b. p. $210-218$. 